\title{
Retracted: Research on Modeling and Dynamic Characteristics of Complex Biological Neural Network Model considering BP Neural Network Method
}

\author{
Advances in Multimedia
}

Received 5 November 2022; Accepted 5 November 2022; Published 22 November 2022

Copyright (c) 2022 Advances in Multimedia. This is an open access article distributed under the Creative Commons Attribution License, which permits unrestricted use, distribution, and reproduction in any medium, provided the original work is properly cited.

\begin{abstract}
Advances in Multimedia has retracted the article titled "Research on Modeling and Dynamic Characteristics of Complex Biological Neural Network Model considering BP Neural Network Method" [1] due to concerns that the peer review process has been compromised.

Following an investigation conducted by the Hindawi Research Integrity team [2], significant concerns were identified with the peer reviewers assigned to this article; the investigation has concluded that the peer review process was compromised. We therefore can no longer trust the peer review process, and the article is being retracted with the agreement of the editorial board.
\end{abstract}

\section{References}

[1] H. Chen, "Research on Modeling and Dynamic Characteristics of complex Biological Neural Network Model Considering BP Neural Network Method," Advances in Multimedia, vol. 2021, Article ID 7646121, 8 pages, 2021.

[2] L. Ferguson, "Advancing Research Integrity Collaboratively and with Vigour," https://www.hindawi.com/post/advancingresearch-integrity-collaboratively-and-vigour/, 2022. 


\title{
Research on Modeling and Dynamic Characteristics of Complex Biological Neural Network Model considering BP Neural
} Network Method

\author{
Hongyan Chen (iD \\ Chongqing Vocational Institute of Engineering, Chongqing 402260, China
}

Correspondence should be addressed to Hongyan Chen; nanxiaoxue@cqvie.edu.cn

Received 5 August 2021; Accepted 18 October 2021; Published 11 December 2021

Academic Editor: Zhendong Mu

Copyright (c) 2021 Hongyan Chen. This is an open access article distributed under the Creative Commons Attribution License, which permits unrestricted use, distribution, and reproduction in any medium, provided the original work is properly cited.

\begin{abstract}
Biological neural network system is a complex nonlinear dynamic system, and research on its dynamics is an important topic at home and abroad. This paper briefly introduces the dynamic characteristics and influencing factors of the neural network system, including the effects of time delay and noise on neural network synchronization, synchronous transition, and stochastic resonance, and introduces the modeling of the neural network system. There are irregular mixing problems in the complex biological neural network system. The BP neural network algorithm can be used to solve more complex dynamic behaviors and can optimize the global search. In order to ensure that the neural network increases the biological characteristics, this paper adjusts the parameters of the BP neural network to receive EEG signals in different states. It can simulate different frequencies and types of brain waves, and it can also carry out a variety of simulations during the operation of the system. Finally, the experimental analysis shows that the complex biological neural network model proposed in this paper has good dynamic characteristics, and the application of this algorithm to data information processing, data encryption, and many other aspects has a bright prospect.
\end{abstract}

\section{Introduction}

Artificial neural network model, as a system of effective data processing, is also a nonlinear dynamic system. According to the continuous research of domestic and foreign scholars, many different models based on neural network algorithms have been developed. Because of the continuous development of artificial neural networks, they have been widely used in many fields. In the actual application process, they have shown that they can effectively solve many complex problems [1-3]. They are also playing an increasingly important role in many industries, such as image recognition. Because artificial neural networks have powerful data information processing and analysis capabilities, domestic researchers have gradually begun to define artificial neural network algorithms for simulation. The multichannel memory resistance pulse-coupled neural network model proposed based on nano-level memory resistance accurately simulates the neural network. The change of the middle connection coefficient can effectively solve the estimation problem of the corresponding parameter in the neural network algorithm process. A 3D memristive HR neuron model based on the overall hidden oscillation is proposed, which can truly reflect the complex dynamic characteristics of brain waves in the neural network [4]. Among them, the state of the turbid system not only is determined by its realtime state but also depends on the initialization state of the system. This characteristic also reflects the characteristics of the biological brain.

Based on the biological neuron's own excitement characteristics, power changes, and the statistical characteristics of biological neuron excitement with different learning coefficients combined with the application of neural network algorithms in real life, this paper uses BP neural network to construct a complex biological neural network model, and the research results show that the learning 
coefficient of the connection weight of the biological neural network has an optimizing effect on the excitement characteristics of the network.

\section{BP Neural Network Algorithm}

Neural networks are often in different states in life, and the corresponding brain wave types are also different. The sine wave signal in brain waves does not exist alone. The lower the frequency, the lower the amplitude, and vice versa [5-7]. Consider the brain. Multifrequency sine wave signals are multiple sine wave signals with different amplitudes, phases, and frequencies. The mathematical expression of the neural network signal is as follows:

$$
x(t)=\sum_{i=1}^{N} A_{i} \sin \left(\omega_{i} t+\varphi_{i}\right),
$$

where $A_{i}, \omega_{i}$, and $\varphi_{i}$ are amplitude, angular frequency, and initial phase, respectively.

In order to facilitate the analysis, this article will simplify it, use the superposition of two sinusoidal signals with different frequencies and initial phases to form a neural network signal, and introduce it into the BP neural network of the biological neural network; the BP neural network is defined as

$$
\begin{aligned}
S(x) & =A_{1} \sin \left(\frac{x}{\varepsilon_{1}}+\varphi_{1}\right)+A_{2} \sin \left(\frac{x}{\varepsilon_{2}}+\varphi_{2}\right) \\
& =A e^{-q|x|}\left[\sin \left(\frac{x \pi}{\varepsilon_{1} e^{-m|x|}}\right)+\sin \left(\frac{2 x \pi}{\varepsilon_{2} e^{-n|x|}+\varphi_{2}}\right)\right] \\
A_{1} & =A_{2}
\end{aligned}
$$

where $x$ is the independent variable, indicating the strength of brain activity; $A, A_{1}$, and $A_{2}$ are amplitudes (the interval is $0 \leq A \leq 1$, in which $A_{1}=A_{2}=A$ ); $q, m$, and $n$ are positive parameters; $\varepsilon_{1}$ and $\varepsilon_{2}$ are two different steepness parameters of the sine function; and $\varphi_{1}$ and $\varphi_{2}$ are the initial phases. When the parameter values are $A=0.3, \varepsilon_{1}=0.04, \varepsilon_{2}=0.02$, $\varphi_{1}=0, \varphi_{2}=\pi / 4=, m=6.8, n=1.8$, and $q=7$, the waveform of the BP neural network is as shown in Figure 1.

It can be seen from Figure 1 that the amplitude and frequency changes of the BP neural network formed by the superposition of two different frequency sine functions are more complicated. Compared with the single-frequency conversion sine function, two sine functions of different frequencies and phases superimpose these functions, making the whole function closer to the biological mechanism of brain waves.

\section{Modeling Problem of Neural Network Model}

This is currently one of the most active directions in computational neuroscience and is an important method and means for studying brain functions [8-10].

There are 3 types of brain networks. One is that the structural network is based on the principles of neuroanatomy and is composed of electrical or chemical connections between neuronal synapses. It is generally determined by anatomy of the subject and MRI. The second is the functional network which describes the neuron clusters (for example, the cortical area). The result of the information generated by the statistical connection relationship between each node is a nondirectional network. The third is the effective network. The effective network describes the interaction or information flow between the nonlinear dynamic behaviors of each node of the layer neural network and is directed toward the network. A reasonable neural network model is extremely important for neuroscientific computing, and it is also an extremely difficult task. Up to now, a lot of modeling work of artificial neural network models has been carried out, but the nature and connection methods of the units are highly artificial. How to combine the characteristics of real biological neural networks and cognitive function requirements, including anatomical connections (considering spatial constraints) and functional connections (considering information exchange and integration), will receive special attention in the future.

In the entire biological neural network system, transmission and conversion of information between neurons are mainly realized by neural keys. According to the combination mechanism of neurons, antenna transmission is divided into electrical antenna and chemical antenna. According to the response characteristics, it is divided into excitatory type and inhibitory type. Since the 1990 s, some theoretical models on the combination of electric cigars and chemical cigars have been established, and various synchronous discharge behaviors of the combined neuronal system have been studied. These results preliminarily reflect the complex phase relationship and the dynamics of information transmission in the neuron clusters and indicate that neuron synchronization plays an important role in the brain's information processing. However, in most neural network modeling, the connection scheme does not consider the spatial distance. In large neuron networks, long-distance connections in space mean high energy consumption. Therefore, realworld models should consider space constraints. In addition, in current research, symmetric and unweighted connection matrices are usually considered, but the actual weighted asymmetric coupling matrix is more reasonable in the interaction of neurons. 
Topology recognition of complex neural networks is also a hot issue, which is the inverse problem of synchronization and control of complex power networks. Current network topology recognition mainly uses adaptive methods to construct auxiliary networks (response networks) and uses adaptive rules to achieve the purpose of identifying network side rights. Because of the large amount of calculation, this method is more difficult for large-scale networks. How to make further use of anti-synchronization, noise, chaos and other novice segments for network topology recognition is a very interesting problem.

\section{The Dynamic Characteristics and Influencing Factors of the Neural Network Model}

The current research studies on neural network synchronization are mainly carried out on complete synchronization problems and phase synchronization of ideal models, but there are few studies on phase synchronization, cluster synchronization, cluster synchronization, and so on, which are closely related to the physiological functions of biological neural network systems $[11,12]$. In particular, how can these synchronizations be combined with the normal and abnormal physiological functions of the biological neural network system? The neural network model has fewer total spatiotemporal dynamics, information transmission, and recognition problems. The dynamic characteristics of neural networks include stability, robustness, functional efficiency, control characteristics, dynamic behavior, spatiotemporal evolution models, etc. These issues are worthy of in-depth study.

Time axis and noise are important factors that cannot be ignored in the actual biological neural network, and for these two factors, the theoretical analysis of scholars often cannot be ignored. Most of them need to use the means based on numerical simulation to explain the problem and analyze whether to form a theoretical system.

In the current research, the electrical combination between nerve elements is considered a lot, but in fact, the information exchange between the neurons, the electrical neurons, and the chemical antennae is carried out at the same time. These play a complementary role in the common rhythm of the overall performance of the network. In addition, the neurons corresponding to these two types should be considered because in the cluster activity of the network, inhibitory neurons and excitatory neurons can cause different dynamic properties. The topological structure and statistical characteristics of neural network models are closely related to neurocognitive functions. What is important is the small-world nature of the brain network. It has been found that we only understand the first step of its complex network structure and dynamic behavior. The development of network science theory requires the establishment of more measurements of network topology characteristics, through experimental measurements, to study the new relationship with the dynamic behavior of neural network models.

\section{Complex Biological Neural Network Model Based on BP Neural Network}

The function with biological characteristics designed in this paper is combined with the original BP neural network as its nonlinear BP neural network. The biological neural network model can be described as

$$
\frac{\mathrm{d} x_{j}}{\mathrm{~d} t}=-x_{j}+a_{j} f\left(x_{j}\right)+\sum_{k=i, k \neq j}^{n} A_{j k} f\left(x_{k}\right)+\sum_{k=i, k \neq j}^{n} S_{j k} x_{k}+I_{j},
$$

where $a_{j}, A_{j k}$, and $S_{j k}$ are constants. When $a_{j}, A_{j k}$, and $f\left(x_{j}\right)$ take some specific values, it can present chaos with rich dynamic characteristics. Let the biological parameters of the sixth-order biological neural network be

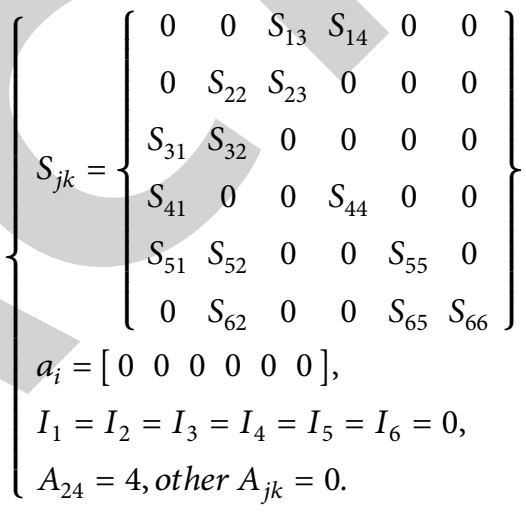

Then, this BP neural network complex biological neuron model can be described as

$$
\left\{\begin{array}{l}
\dot{x}_{1}=S_{13} x_{3}+S_{14} x_{4} \\
\dot{x}_{2}=S_{22} x_{2}+S_{23} x_{3}+A_{24} f\left(x_{4}\right) \\
\dot{x}_{3}=S_{31} x_{1}+S_{32} x_{2} \\
\dot{x}_{4}=S_{41} x_{1}+S_{44} x_{4} \\
\dot{x}_{5}=S_{51} x_{1}+S_{52} x_{2}+S_{55} x_{5} \\
\dot{x}_{6}=S_{62} x_{2}+S_{65} x_{5}+S_{66} x_{6}
\end{array}\right.
$$

Among them,

$$
\begin{aligned}
& f\left(x_{4}\right)=A c e^{-q\left|x_{4}\right|}\left[\sin \left(\frac{x_{4} \pi}{\varepsilon_{1} e^{-m\left|x_{4}\right|}}\right)+\sin \left(\frac{2 x_{4} \pi}{\varepsilon_{2} e^{-n\left|x_{4}\right|}}+\varphi\right)\right]+g\left(x_{4}\right), \\
& g\left(x_{4}\right)=\frac{1}{2}\left(\left|x_{4}+1\right|-\left|x_{4}-1\right|\right) .
\end{aligned}
$$

This article takes several representative parameters $c, q$, and $n$ in the BP neural network to analyze the influence of the BP neural network on the biological neural network. Among them, $c$ represents the proportional coefficient of the BP neural network, $q$ is the parameter that controls the amplitude $A$, and $n$ is the parameter that controls the frequency change. As the parameters $c, q$, and $n$ change, the 


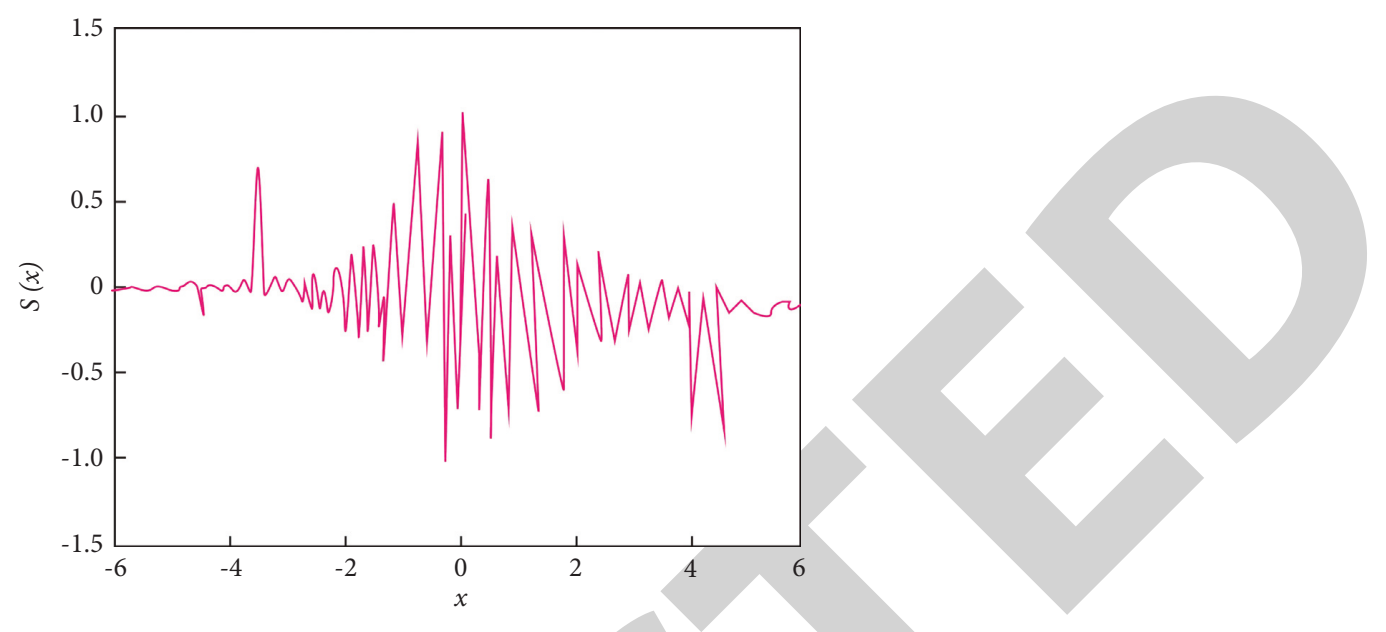

FIGURE 1: BP neural network function image.

system will be in a different state. Fix the initial conditions $(0.1,0.2,0.3,0.4,0.5,0.6)$ and let the parameters $c, q$, and $n$, respectively, change within a certain interval; the values of other parameters are listed in Table 1, and the bifurcation diagram and Lyapunov exponent spectrum of the corresponding system are shown in Figure 2.

\subsection{The Influence of BP Neural Network Parametern on System} Dynamics. When $n$ changes in the interval $[0,5]$, the values of $c$ and $q$ are $c=0.25$ and $q=-1$, and the remaining parameters are listed in Table 1 . The branch diagram and Lyapunov index spectrum of the system are shown in Figure 3.

In the BP neural network, $n$ is the parameter that controls the frequency change. It can be seen from Figure 3 that as the parameter $n$ increases, the system becomes chaotic. Therefore, in the frequency range of $n>3.2$, the system does not appear to be complicated.

5.2. The Influence of BP Neural Network Parameter $c$ on Dynamic Characteristics. When $c$ changes in the interval [0, $1]$, the values of $q$ and $n$ are $q=-1$ and $n=2.5$, and the remaining parameters are shown in Table 1 . The branch diagram and Lyapunov index spectrum of the system are shown in Figure 4.

It can be seen from Figure 4 that the system branches periodically through periodic orbits and becomes a chaotic state. The parameter $c$ is the proportional coefficient of the BP neural network. When $c$ is larger, the proportion of the BP neural network function in the biological neural network is also larger. As the parameter $c$ gradually increases, the chaotic mapping interval of the variable $x$ (amplitude) gradually increases. When $c$ runs to 0.51 and 0.7 , the chaotic mapping interval suddenly increases. At this time, the attractor of the system becomes more complicated. This phenomenon also verifies that the larger the parameter $c$. The larger the proportion of the neural network function, the larger the chaotic mapping interval of system variables, and its dynamic characteristics are more varied. Its typical chaotic attractor is shown in Figure 5.

5.3. The Influence of BP Neural Network Parameter $q$ on Dynamic Characteristics. When $q$ changes in the interval $[-1.5,0.5]$, the values of $c$ and $n$ are 0.25 and 2.5 , and the remaining parameters are listed in Table 1 . The branch diagram and Lyapunov index spectrum of the system are shown in Figure 6.

In the BP neural network, $q$ is the parameter that controls the amplitude $A$. It can be seen that as the parameter $q$ increases, the system gradually evolves from chaotic to periodic through several inverse period-doubling bifurcation windows, and there are many times in the whole evolution process. The switching between periodic orbits and chaotic orbits puts a certain limit on the amplitude of the system's BP neural network. Several typical phase diagrams of the $x-y$ phase plane attractor varying with $q$ are shown in Figure 7 .

It can be seen from Figures 3, 4, and 6 that when the system changes with the parameters $c, q$, and $n$, the Lyapunov exponents of the generated chaotic attractors are all large. When the parameter $c$ changes, the maximum Lyapunov exponent is 2.5214, and when the parameter $q$ changes, the maximum Lyapunov exponent is 2.0653. The large Lyapunov exponent reflects the degree of separation between the orbits of the system when the initial value changes, that is, it has better initial sensitivity. This shows that the system will produce more complex chaotic dynamics under the action of BP neural network. 
TABle 1: The values of other parameters when the parameters $c, q$, and $n$ take different values.

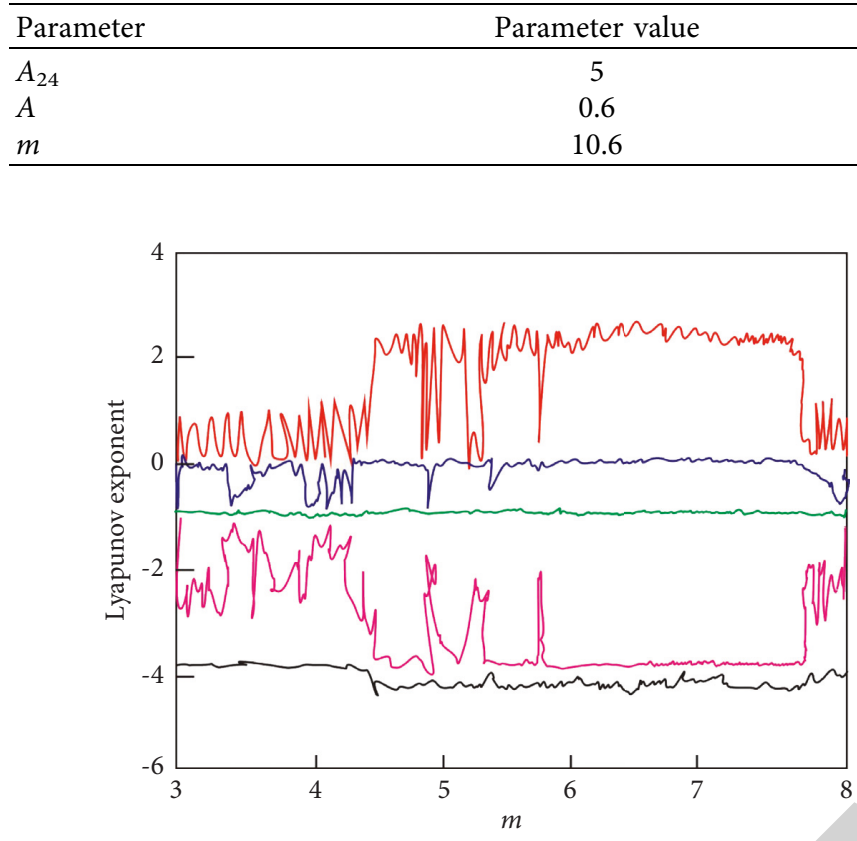

(a)

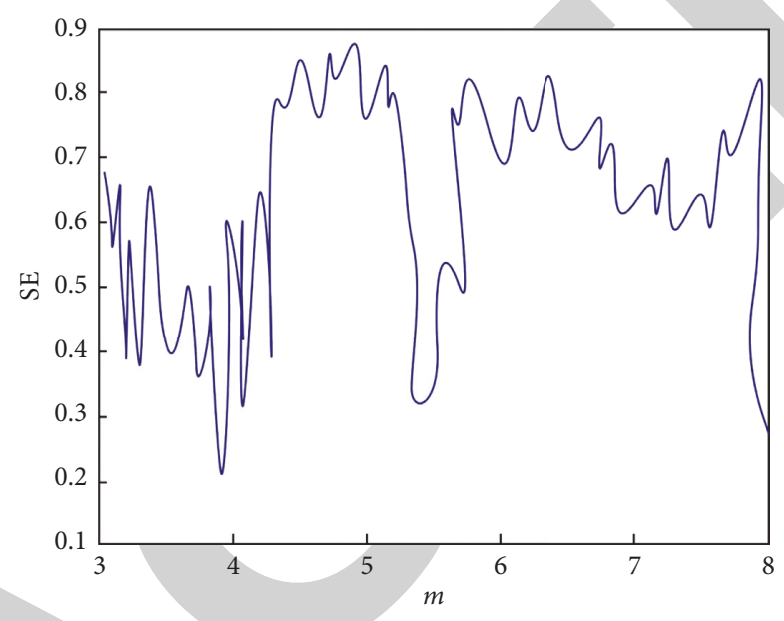

(b)

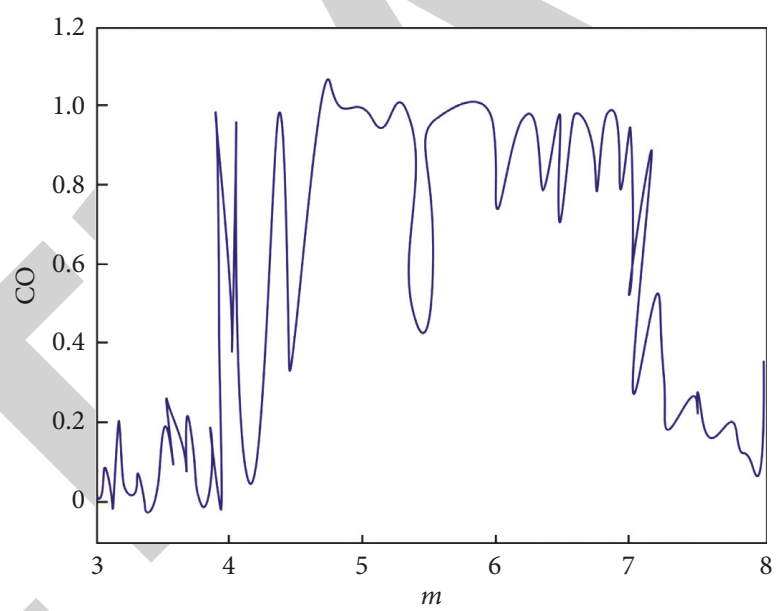

(c)

Figure 2: Two-dimensional complexity of the system: (a) Lyapunov exponential spectrum; (b) SE diagram; (c) C0 diagram.

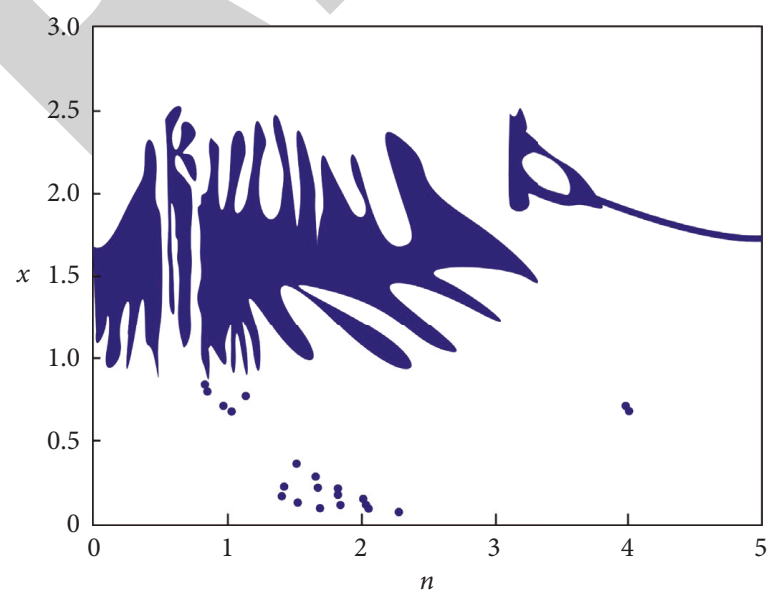

(a)

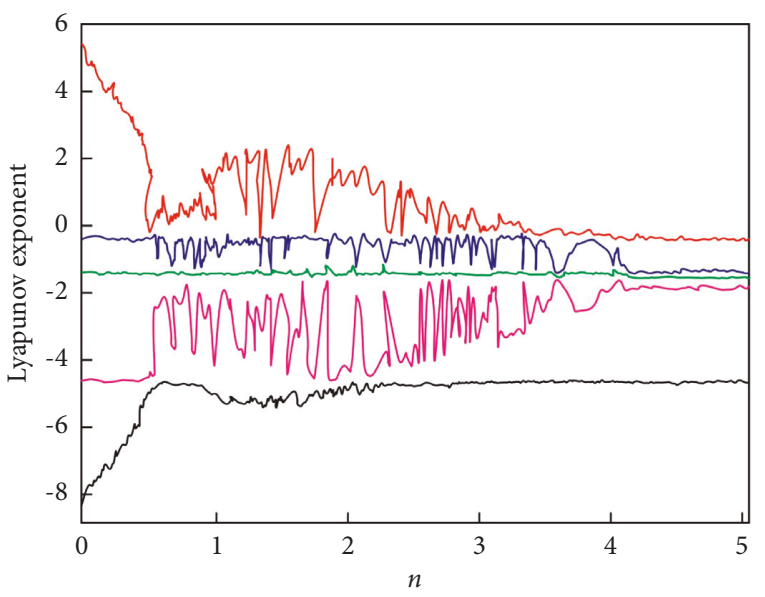

(b)

FIgURE 3: The system shows the branch diagram and Lyapunov index spectrum that vary according to the parameter $n$ : (a) branch diagram; (b) Lyapunov exponent spectrum. 


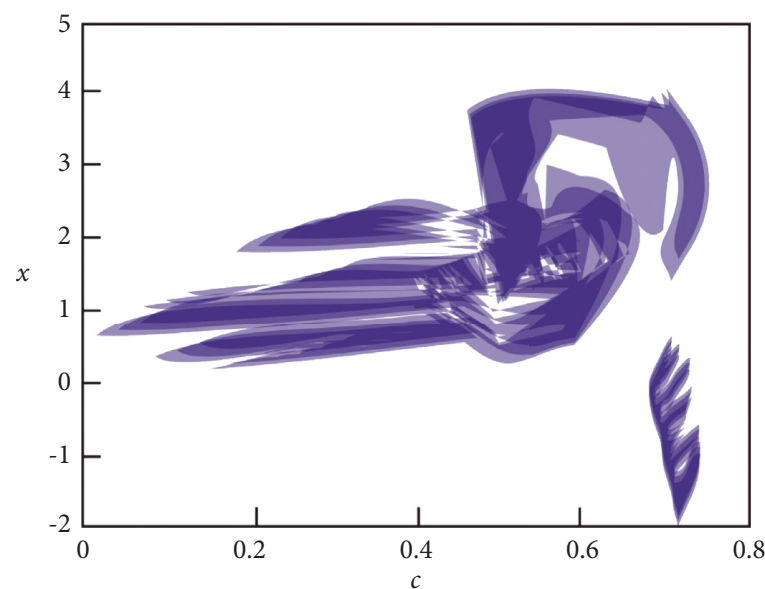

(a)

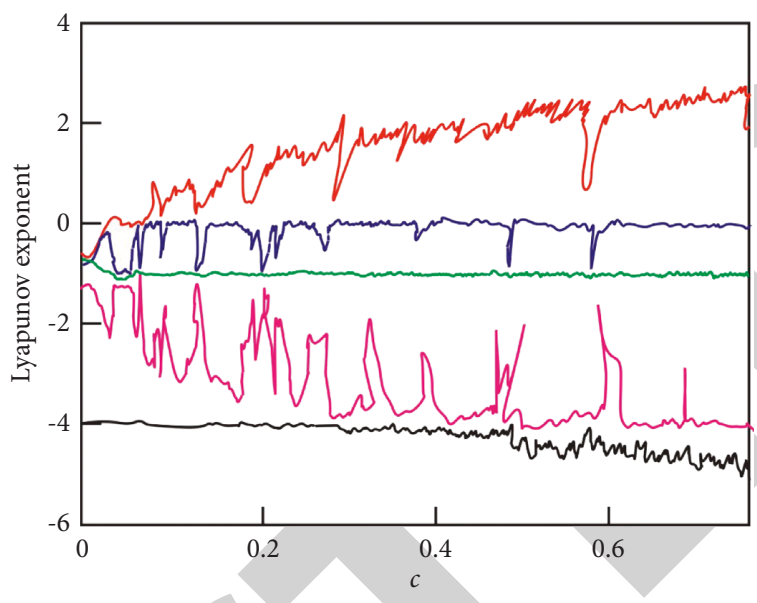

(b)

FIGURE 4: The system shows the branch diagram and Lyapunov index spectrum that vary according to parameter $c$ : (a) bifurcation graph and (b) Lyapunov exponent spectrum of the system with the parameter $c$.

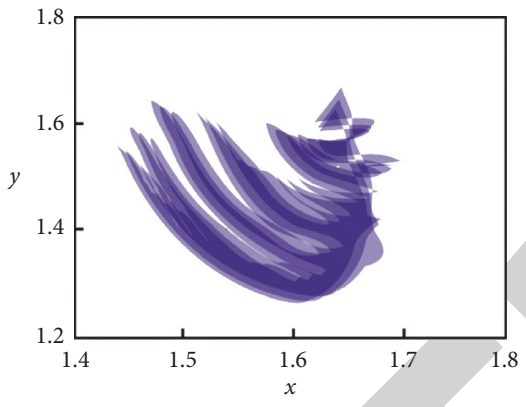

(a)

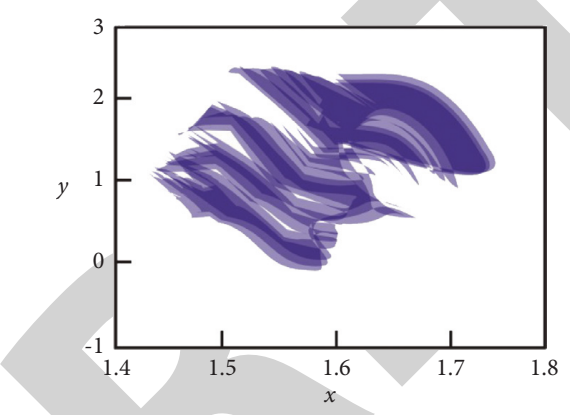

(d)

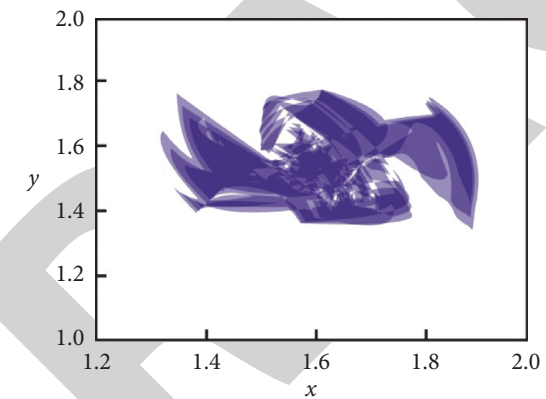

(b)

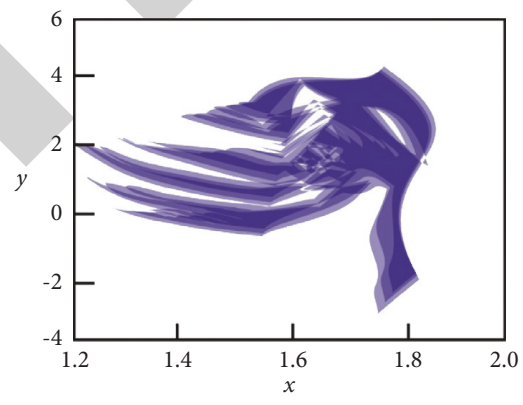

(e)

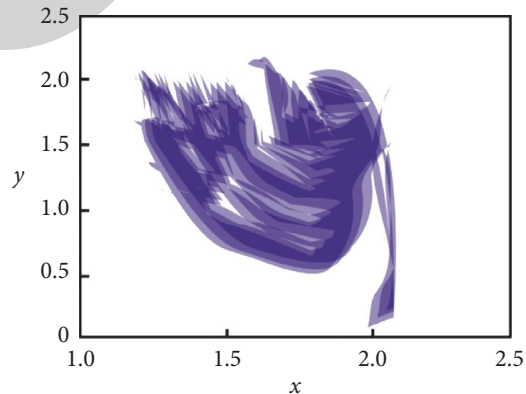

(c)

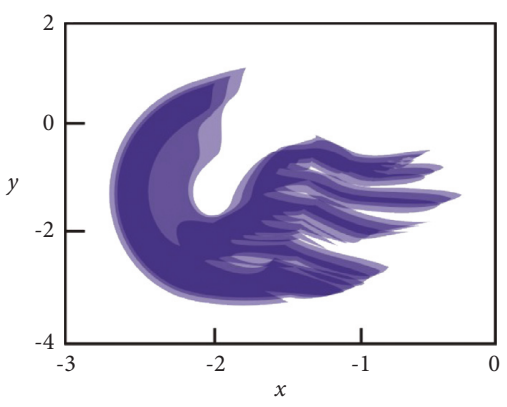

(f)

Figure 5: Phase diagram of the system changing with parameter $c$ : (a) $c=0.07$; (b) $c=0.117$; (c) $c=0.25$; (d) $c=0.51$; (e) $c=0.532$; (f) $c=0.72$.

\section{Analysis of Dynamic Characteristics of Complex Biological Neural Network Model}

The system setting parameters $A_{24}=6, A=0.5, c=0.24$, $m=5.6, n=2.7, q=-1, \varepsilon_{1}=0.05, \varepsilon_{2}=0.04$ and $\varphi=-\pi / 4$. The changes in the initial conditions $[0.1, y(0), 0.3,0.4,0.5,0.6]$ are shown in Figure 8 for the Lyapunov exponent spectrum and branch diagram of the system.

It can be seen that the system is in a chaotic state most of the time, and its trajectory is roughly divided into three parts. The first part is in the state of switching between periodic orbits and chaotic orbits; the second and third parts are in a chaotic state, and its attractor type and shape change accordingly, as shown in Figure 9. When $y(0)$ is in the interval $[-2,-1]$, the system is in the periodic state most of the time, but there are many times of switching between the periodic orbit and the chaotic orbit. When $y(0)$ is in the interval $[-1,2]$, the system is in the chaotic state, but when $y$ ( 0 When) is at 0.7 , the chaos are as shown in Figure 8.

13, 14 The traditional initial value sensitivity generally refers to the same. In a chaotic attractor, the chaotic orbits are separated rapidly due to the small disturbance of the 


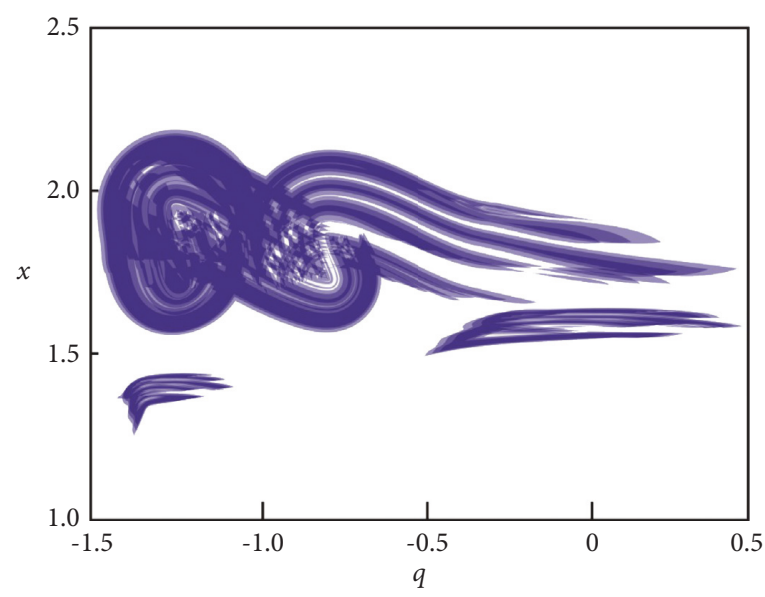

(a)

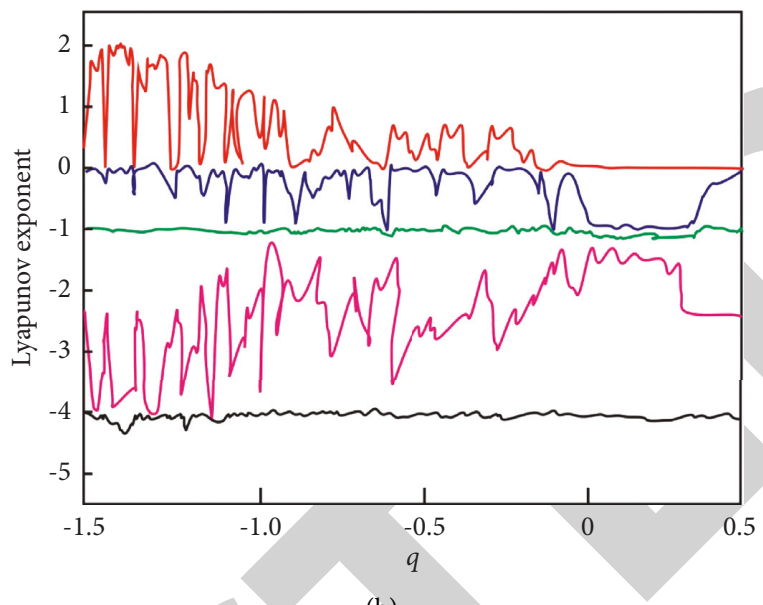

(b)

FIGURE 6: The system shows the branch diagram and Lyapunov index spectrum varying with the parameter $q$ : (a) branch diagram; (b) Lyapunov exponent spectrum.

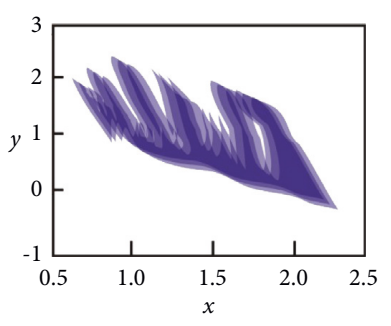

(a)

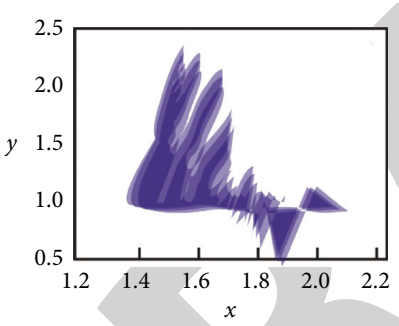

(b)

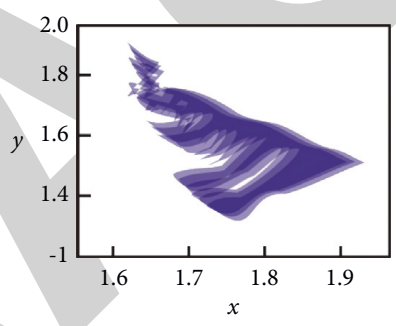

(c)

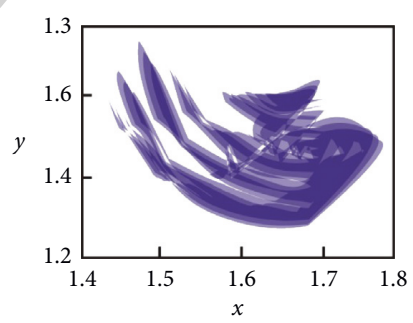

(d)

Figure 7: The phase diagram of the system changing with the parameter $q$ : (a) $q=-1.25$; (b) $q=-0.74$; (c) $q=-0.35$; (d) $q=-0.24$.

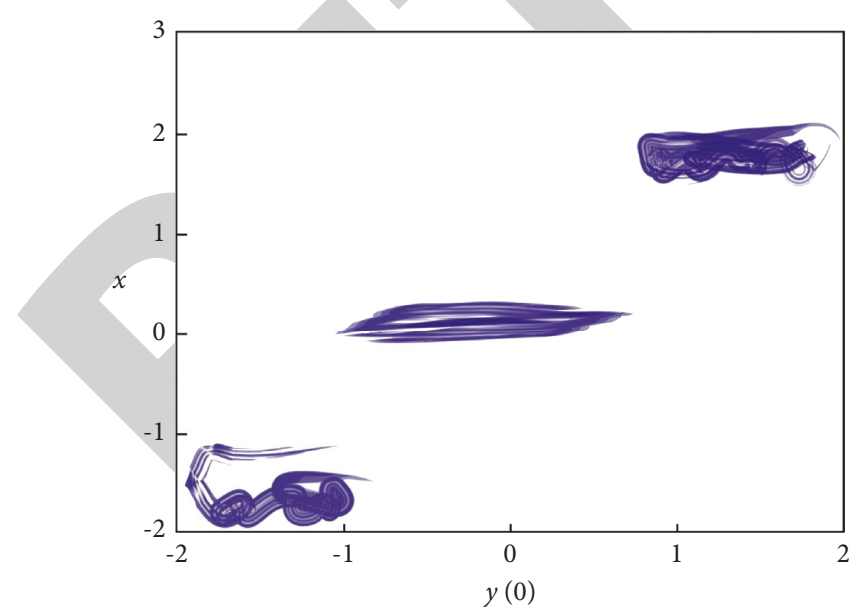

(a)

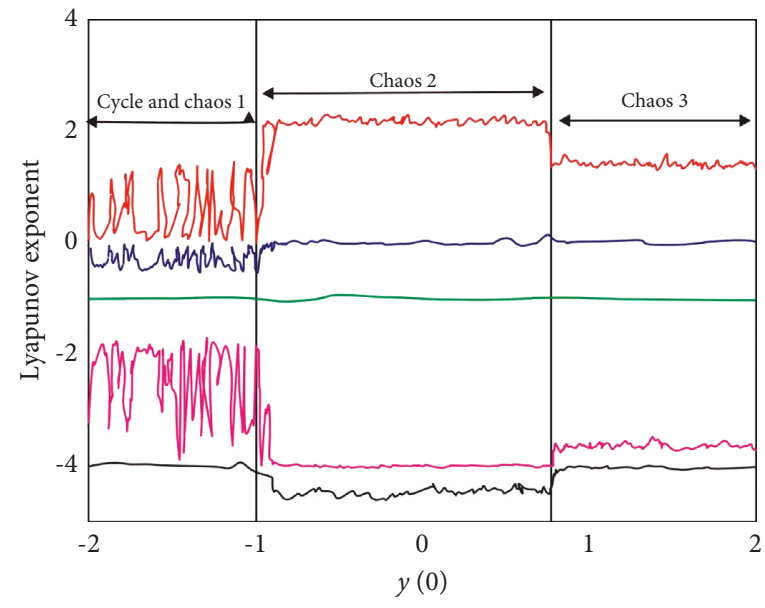

(b)

FIGURE 8: The branch graph and Lyapunov exponent spectrum of the system with the initial value $y$ (0): (a) branch graph; (b) Lyapunov exponent spectrum.

initial value. It has the characteristics of system dynamics, but the characteristics are different. Therefore, there are two types of coexisting attractors: different attractors with the same dynamic behavior, called homogenous coexistence attractors, and different attractors with different dynamic behaviors, called heterogeneous coexisting attractors. If the 


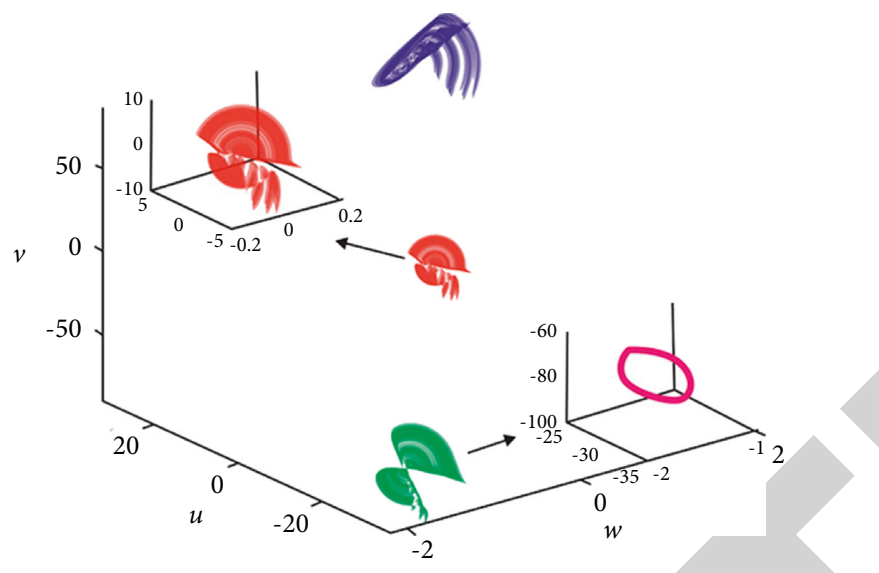

Figure 9: Phase diagram of the system changing with the initial value $y(0)$.

chaotic system has a coexisting attractor, the chaotic system is more sensitive to the initial value and has more complex dynamic behavior, and the system has better application value in information encryption and secure communication.

\section{Conclusions}

In this paper, the BP neural network algorithm is used in the construction of complex biological neural network models. The neural network model can generate good dynamic characteristics, which are mainly composed of different types of mixed attractors, periodic attractors, and different types of coexisting attractors. The experimental analysis results show that the complex biological neural network model constructed in this paper has good performance. Kinetic characteristics.

\section{Data Availability}

The data used to support the findings of this study are available from the corresponding author upon request.

\section{Conflicts of Interest}

The author declares that there are no conflicts of interest.

\section{References}

[1] W. Lotter, G. Kreiman, and D. Cox, “A neural network trained to predict future video frames mimics critical properties of biological neuronal responses and perception," Neurons and Cognition, vol. 49, no. 10, pp. 925-936, 2018.

[2] S. T. Hill, K. Rachael, T. Amy, M. Erich, D. Padideh, and D. A. Hendrix, "A deep recurrent neural network discovers complex biological rules to decipher rna protein-coding potential," Nuclc Acids Research, vol. 23, no. 10, pp. 2626-2682, 2018.

[3] K. K. Mineeja and R. P. Ignatius, "Lévy noise-induced neardeath spikes and phase transitions of a biological neural network," Nonlinear Dynamics, vol. 99, no. 4, pp. 3265-3283, 2020.

[4] X. Li, Q. Chen, and F. Xue, "Biological modelling of a computational spiking neural network with neuronal avalanches," Philosophical Transactions. Series A, Mathematical, Physical, and Engineering Sciences, vol. 375, no. 9, p. 417, 2017.

[5] U. Hasson, S. A. Nastase, and A. Goldstein, "Direct fit to nature: an evolutionary perspective on biological and artificial neural networks," Neuron, vol. 105, no. 3, pp. 416-434, 2020.

[6] S. Wang, K. Fan, N. Luo, Y. Cao, and L. You, "Supplementary info for massive computational acceleration by using neural networks to emulate mechanism-based biological models.pdf," Nature Communications, vol. 10, no. 1, pp. 1-9, 2019.

[7] J. Gong and M. Yang, "Evolutionary fault tolerance method based on virtual reconfigurable circuit with neural network architecture," IEEE Transactions on Evolutionary Computation, vol. 22, no. 6, pp. 949-960, 2018.

[8] M. Haesemeyer, A. F. Schier, and F. Engert, "Convergent temperature representations in artificial and biological neural networks," Neuron, vol. 55, no. 14, pp. 429-435, 2019.

[9] K. K. Mineeja and R. P. Ignatius, "Spatiotemporal activities of a pulse-coupled biological neural network," Nonlinear Dynamics, vol. 42, no. 3, pp. 1088-1093, 2018.

[10] A. Yp, A. Hl, C. Tsb, and B. Hla, "Exploring biological motion perception in two-stream convolutional neural networks," Vision Research, vol. 178, pp. 28-40, 2021.

[11] M. Sadeghassadi, C. J. B. Macnab, B. Gopaluni, and D. Westwick, "Application of neural networks for optimalsetpoint design and MPC control in biological wastewater treatment," Computers and Chemical Engineering, vol. 115, no. 12, pp. 150-160, 2018.

[12] B. Szalkai, V. Grolmusz, and Vince, "Seclaf: a webserver and deep neural network design tool for hierarchical biological sequence classification," Bioinformatics, vol. 34, no. 14, pp. 2487-2489, 2018.

[13] C. J. Spoerer, M. C. Patrick, and K. Nikolaus, "Recurrent convolutional neural networks: a better model of biological object recognition," Frontiers in Psychology, vol. 152, no. 5, pp. 27-35, 2017.

[14] X. Li, Q. Chen, and F. Xue, "Biological modelling of a computational spiking neural network with neuronal avalanches," Philosophical Transactions of the Royal Society: Mathematical, Physical, and Engineering Sciences, vol. 42, no. 24, pp. 9482-9488, 2017. 